\title{
Fluid-structure interaction between trains and noise-reduction barriers: numerical and experimental analysis
}

\author{
M. Belloli, B. Pizzigoni, F. Ripamonti \& D. Rocchi \\ Mechanical Engineering Department, Politecnico di Milano, Italy
}

\begin{abstract}
This paper deals with the fluid-structure interaction related to the transit of railway vehicles in proximity to noise-reduction barriers. During train transit a pressure wave is generated and consequently, due to the barrier's large surface area, non-negligible loads on the support elements arise. This problem applies in particular to high-speed railway lines and becomes critical when the train-barrier gap is small. Moreover, the presence of lateral wind can increase the loads acting on the structures, influencing the design of the support uprights. The problem has been numerically and experimentally evaluated. A preliminary study consisted of Computational Fluid Dynamics (CFD) simulations, reproducing the train and barriers geometries and the relative motion with the "sliding mesh" technique. The conditions of both the absence and presence of lateral wind were investigated. The pressure distribution along the barriers was analysed for different conditions. Then, experimental tests were performed in the wind tunnel at the Politecnico di Milano. Different wind speeds and exposure angles were considered in order to define lateral wind contributions. The pressure on the barriers was calculated at several points by means of pressure taps. Finally, the results obtained were compared with the data collected during an experimental campaign performed on the Rome-Naples high-speed railway line.
\end{abstract}

Keywords: train transit, noise reduction barriers, CFD, wind tunnel, pressure distribution.

\section{CFD code study}

A Computational Fluid Dynamics (CFD) code was used to perform simulations of the flow field induced by the train transit in the proximity of a noise reduction 
barrier [1-3], in still air or with lateral wind. Simulations were performed by means of a commercial code on a three dimensional domain, using an unsteady approach and the "sliding mesh" technique. The computational domain was split into two parts, one containing the noise reduction barrier and the surroundings, which is considered at rest, and one containing a small part of air around the train, moving together with the train at the train velocity. The moving domain slides on the still domain along interface planes where the grid points belonging to the different sub-domains do not coincide and their relative position changes with time.

Figure 1 shows a sketch of the computational domain at rest and only the train walls of the moving domain, at the instant of time when the train's head reaches the midpoint of the noise reduction barrier. The boundary conditions adopted in the simulations are also illustrated in the same figure.

Two different sets of boundary conditions were considered to perform simulations with and without lateral wind.

For the simulations without lateral wind, atmospheric pressure conditions were assumed at the pressure inlet and outlet boundaries of the domain and the computed flow field was due to the relative motion between the train and the still barrier.

For the simulations with lateral wind, a static pressure value was prescribed at pressure inlet and at pressure outlet, so that the pressure difference originated a flow in the computational domain, directed transversally with respect to the train direction of motion.

No slip conditions were used for the ground wall, while symmetry conditions were assumed for all the other boundaries in both simulations i.e. with and without lateral wind.

The reference frame has the $\mathrm{x}$-axis aligned with train moving direction, the $\mathrm{y}$ axis aligned with the lateral wind, if any, and the vertical z-axis directed upwards.

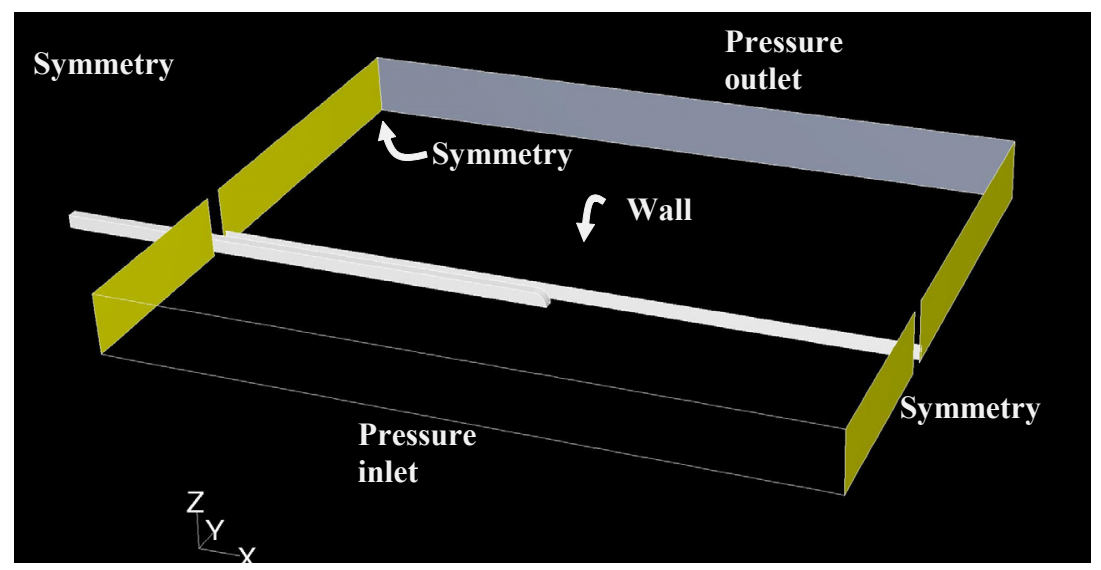

Figure 1: Computational domain, boundary conditions and reference frame. 
The train geometry is reproduced by a simple prism representing the volume of the whole convoy, neglecting all the particulars in the under floor region (bogies and auxiliary parts) and on the roof, keeping account of the variation of the train head section only. The length of the train is chosen in order to prevent the train tail from entering the domain, since only the effects induced by the train head are considered.

\subsection{Simulations without lateral wind}

Figure 2 shows the pressure time histories computed during a train transit at 300 $\mathrm{km} / \mathrm{h}$ on different points belonging to a vertical section of the noise reduction barrier positioned at $\mathrm{x}=80 \mathrm{~m}$ from the train entrance into the still domain. The considered points are positioned at $\mathrm{z}=0.5 ; 1 ; 1.5 ; 2 ; 2.5 ; 3$ and $4 \mathrm{~m}$ from the top of rail (TOR) level. The overpressure recorded at different heights from the ground is compared, showing how the peak values become lower the higher is the quote from the ground. This reduction is due to the effect of the constraint acted by the barrier on the air trapped between the lateral wall of the train and the barrier itself and moving towards the upper edge of the barrier. An overpressure peak of more than $500 \mathrm{~Pa}$ and a suction peak of almost $-900 \mathrm{~Pa}$ are computed when the train head reaches the investigated section position. Before the overpressure peak a positive pressure is already present, due to the mass of air dragged by train.

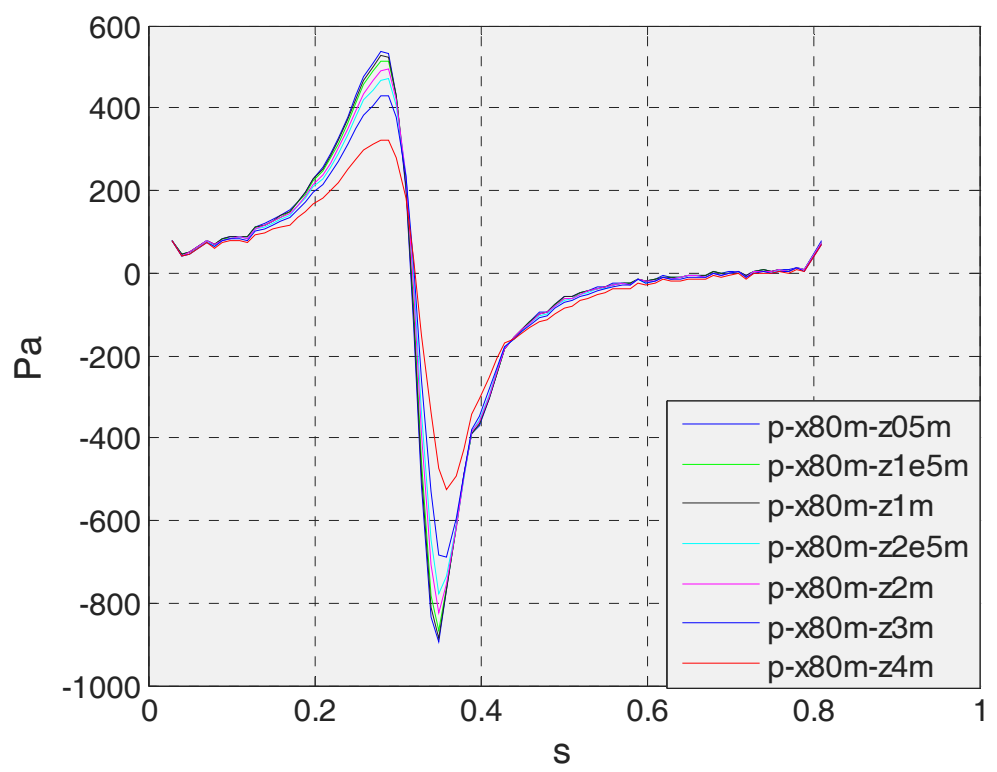

Figure 2: Time histories of the pressure computed by the CFD simulation, at different heights from the ground. 


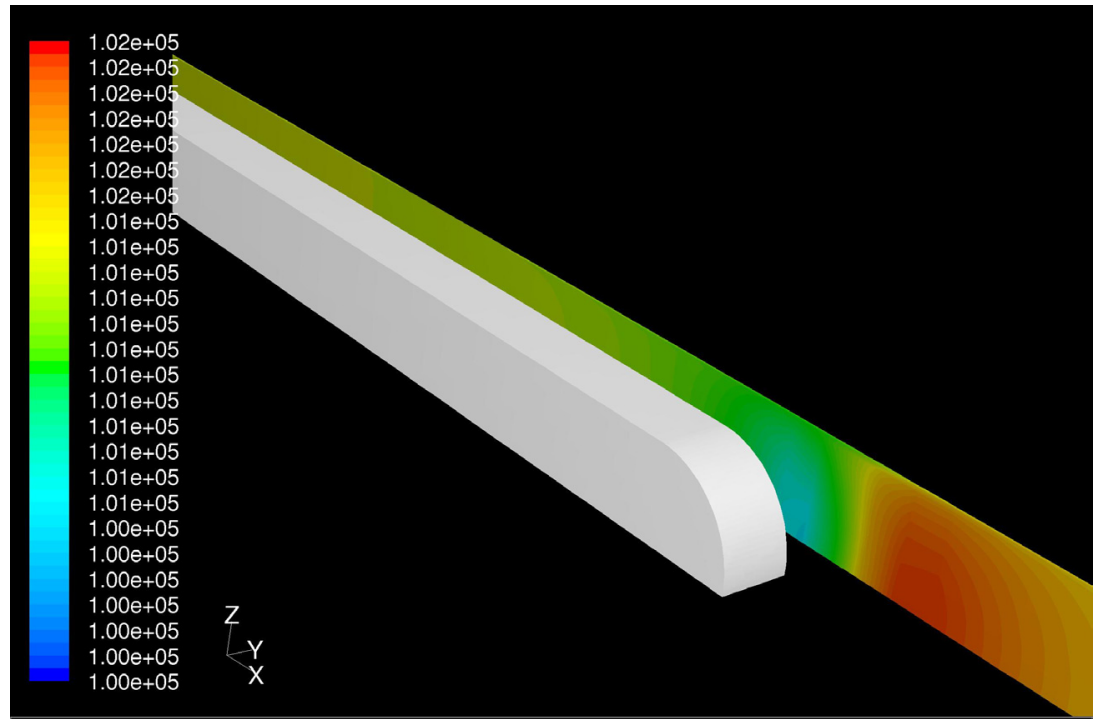

Figure 3: Contour plot of the static pressure on the noise reduction barrier.

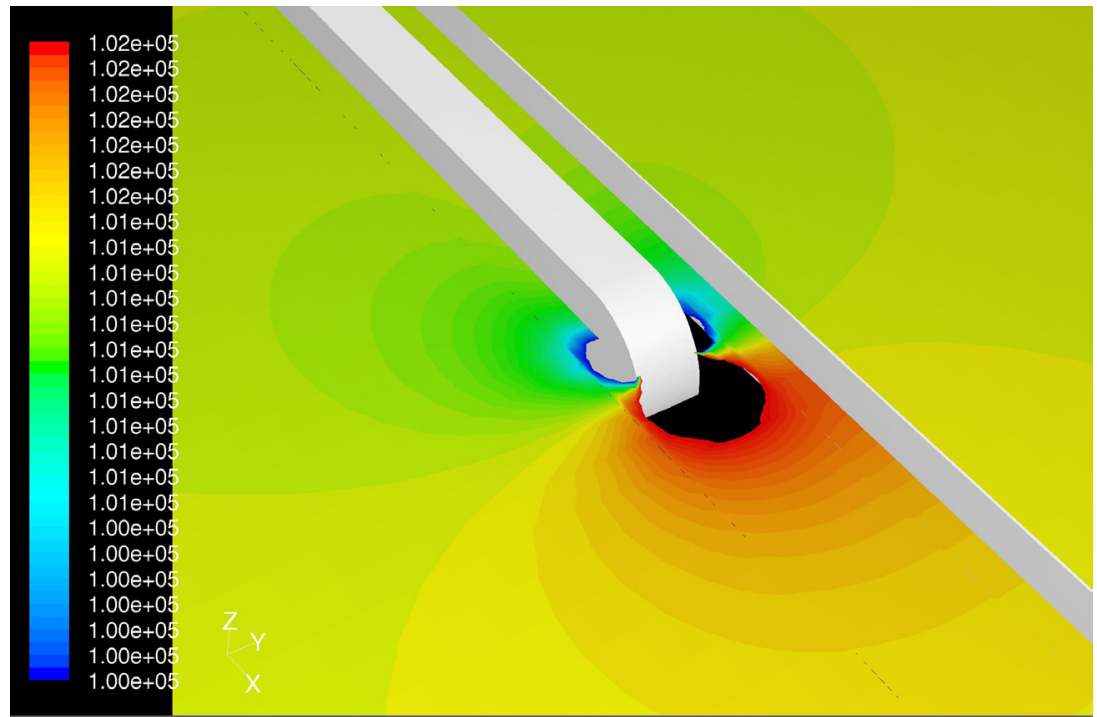

Figure 4: Static pressure contour plot.

Figure 3 shows the contour plot of the static pressure field on the noise reduction barrier. The red zone is characterised by overpressure and is positioned just in front of the train head; the blue one is characterised by suction and is positioned aside the train head. 
The reduction of pressure moving upwards from the ground level can also be appreciated as well as the extension of the positive pressure zone in front of the train.

In Figure 4, a contour plot of the static pressure on an horizontal plane positioned at a height of $2.5 \mathrm{~m}$ from the TOR is illustrated, describing the lack of symmetry in the pressure field around the train as induced by the barrier. The air is compressed by the train head on the barrier, giving rise to the overpressure zone on the barrier and to a flow in the channel between the lateral wall of the train and the barrier itself.

The pressure time histories computed along the barrier in different sections along the $\mathrm{x}$-axis at $1 \mathrm{~m}$ height from the TOR are compared in Figure 5. The considered sections are equally spaced along the $\mathrm{x}$ axis (train direction) every 10 $\mathrm{m}$, starting from $\mathrm{x}=70 \mathrm{~m}$. The time history trends are very similar and a time lag separates the different results, according to the time required by the train to reach the different positions along the line. An increase both in the positive and the negative peak values is visible, basically due to the unsteadiness of the simulation, which continues to change the boundary conditions while the train is moving.

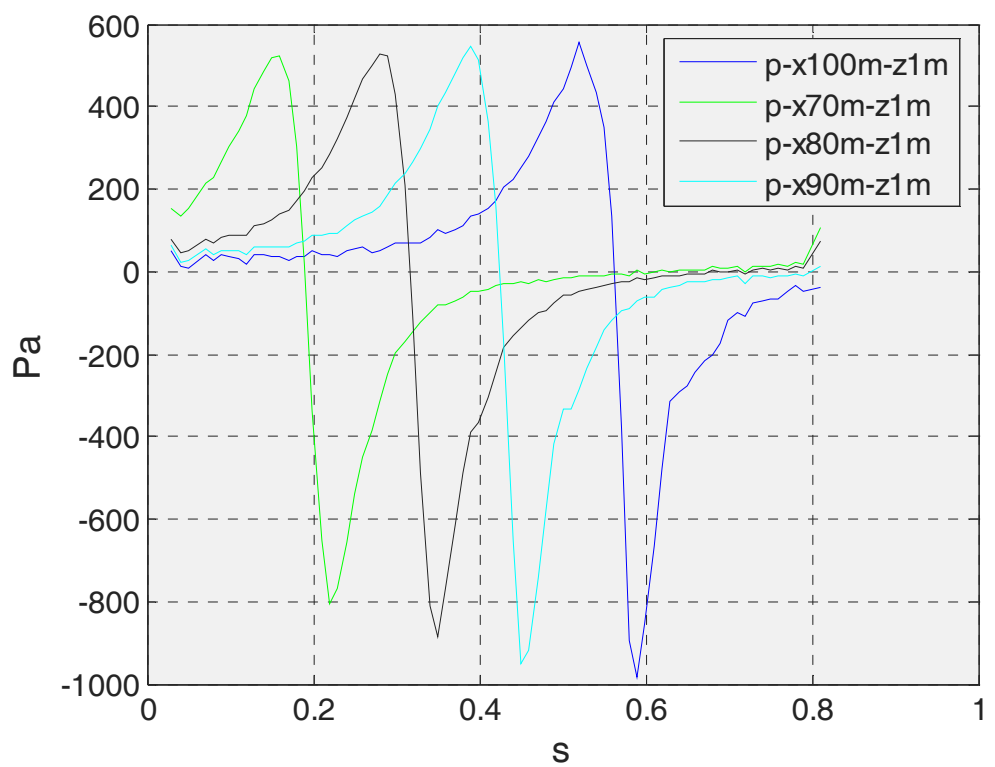

Figure 5: Pressure time histories on different sections along the barrier at $1 \mathrm{~m}$ from the TOR.

\subsection{Simulations with lateral wind}

Figure 6 shows the net pressure time histories, computed on the same points of the $x=80 \mathrm{~m}$ section considered in Figure 2, for a train speed of $300 \mathrm{~km} / \mathrm{h}$ and a 
54 Fluid Structure Interaction $\mathrm{V}$

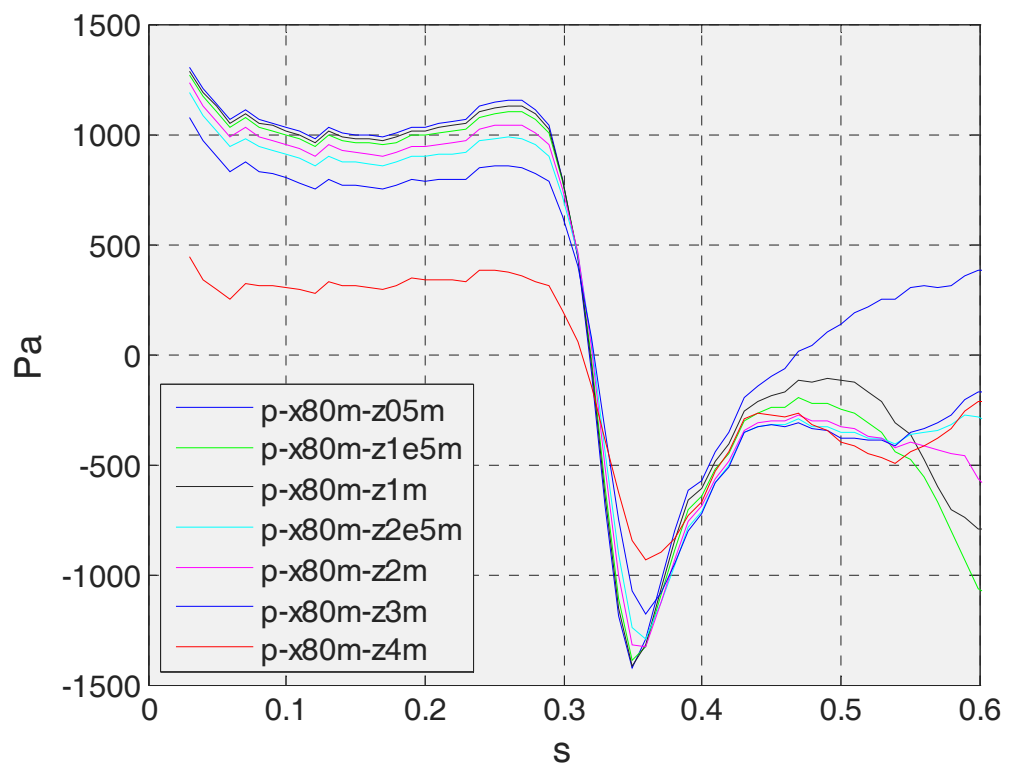

Figure 6: Net pressure time histories at different heights on the barrier section at $\mathrm{x}=80 \mathrm{~m}$.

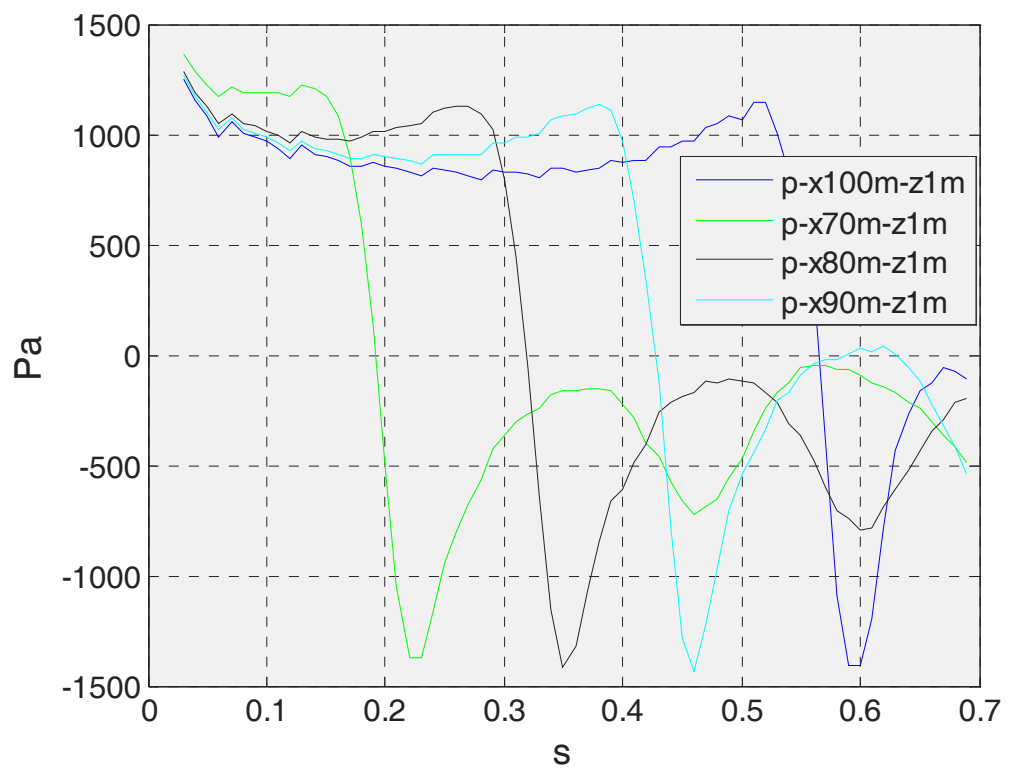

Figure 7: Net pressure time histories on different points along the noise reduction barrier at $1 \mathrm{~m}$ height from TOR. 
lateral wind of $30 \mathrm{~m} / \mathrm{s}$. In this case, the barrier, which is subjected to the wind loads and shows a positive mean value of the net pressure between upwind and downwind surfaces, experiences a reduction of pressure, taking advantage of the wind shielding effect during the train transit. The peak values of the negative pressure are slightly higher than those reached during the simulation without lateral wind. An almost null pressure condition is reached after the negative peak at the considered barrier section, due to the wind shielding effect of the train body.

An analysis of the pressure distribution along the barrier is illustrated in Figure 7, where the net pressure time histories at the same positions considered without lateral wind in Figure 5 are compared. The phenomenon repeats itself in all positions, considering the time delay, and an oscillating negative pressure trend appears after the negative peak is reached.

\section{Wind tunnel tests}

Wind tunnel tests [4] were performed in a 1:10 scale to determine the pressure distribution on the noise reduction barrier during the train transit. Figure 8 shows the complete wind tunnel set-up installed in the boundary layer test section of the Politecnico di Milano wind tunnel. A 1:10 scale model of the EMUV250 train is installed on a flat ground scenario, with a splitter plate at the height of $0.3 \mathrm{~m}$ from the wind tunnel floor, so as to consider the incoming wind to have a block vertical profile of the mean velocity.

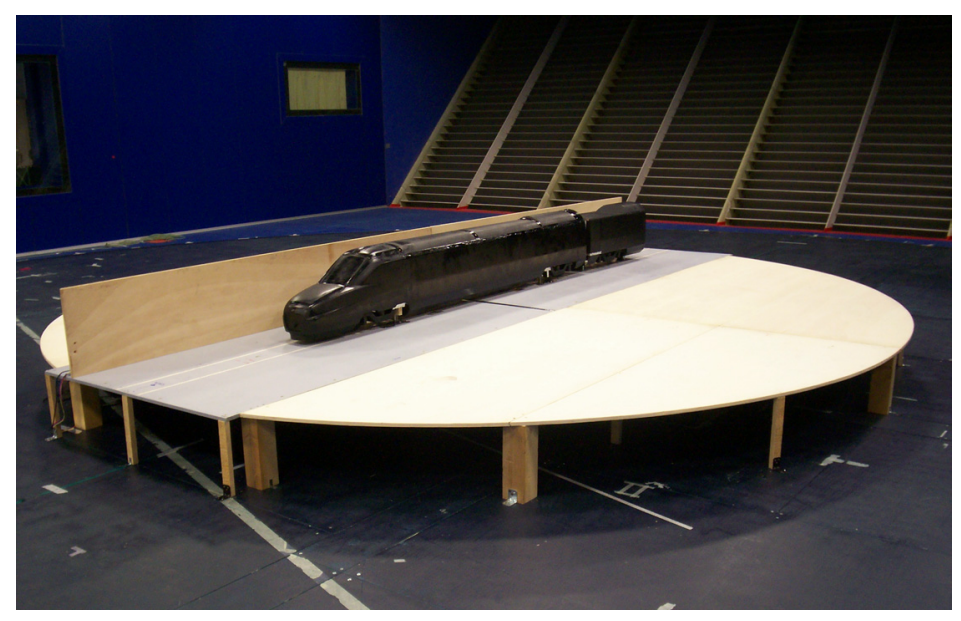

Figure 8: $\quad$ Test set-up installed on the wind tunnel turntable.

To measure the pressure distribution due to the train transit, the noise reduction barrier was instrumented with 32 pressure taps, disposed according to the sketch reported in Figure 9. As can be noted, the pressure taps distribution is more refined close to the train head, positioned at $1.68 \mathrm{~m}$ from the barrier upwind vertical edge (a vertical line is drawn at the train head position in 


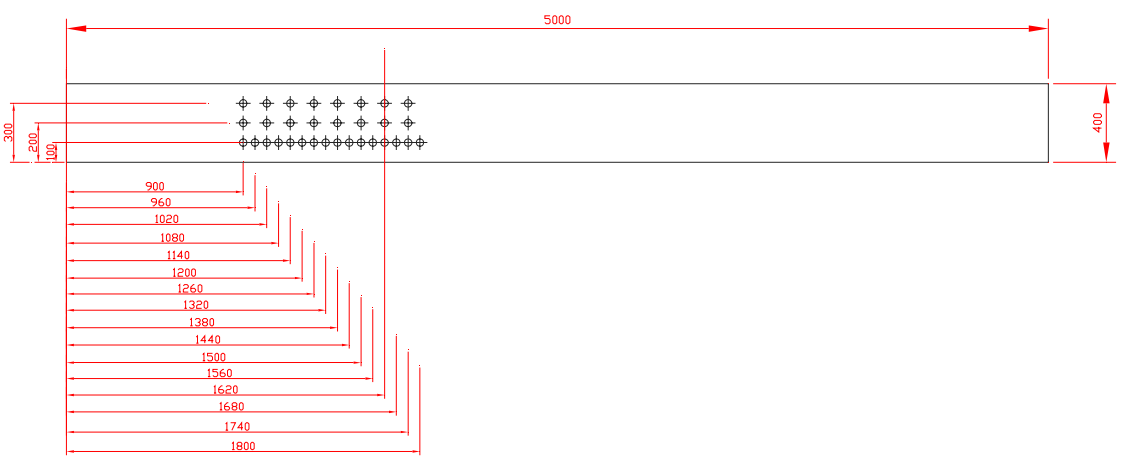

Figure 9: Pressure tap positions on the barriers and train head position.

Table 1: $\quad$ Yaw angles and lateral wind speed at full scale.

\begin{tabular}{|c|c|c|}
\hline Angle & Train velocity & Lateral wind speed \\
\hline $0^{\circ}$ & $83 \mathrm{~m} / \mathrm{s}$ & $0 \mathrm{~m} / \mathrm{s}$ \\
\hline $10^{\circ}$ & $83 \mathrm{~m} / \mathrm{s}$ & $14.6 \mathrm{~m} / \mathrm{s}$ \\
\hline $20^{\circ}$ & $83 \mathrm{~m} / \mathrm{s}$ & $30 \mathrm{~m} / \mathrm{s}$ \\
\hline $30^{\circ}$ & $83 \mathrm{~m} / \mathrm{s}$ & $48 \mathrm{~m} / \mathrm{s}$ \\
\hline
\end{tabular}

Figure 9). The pressure taps have been installed at three different height levels, corresponding to $1 \mathrm{~m}, 2 \mathrm{~m}$ and $3 \mathrm{~m}$ at full-scale size. Each pressure tap is connected to a high frequency pressure scanner. The pressure signals have been acquired at a sampling rate of $100 \mathrm{~Hz}$.

To simulate lateral wind conditions the angle between the wind tunnel incoming wind and the train direction was changed. Four different yaw angles have been analysed in particular, respectively equal to $0^{\circ}, 10^{\circ}, 20^{\circ}$ and $30^{\circ}$. The $0^{\circ}$ deg test corresponds to the absence of lateral wind. Assuming a train speed of $300 \mathrm{~km} / \mathrm{h}$,

Table 1 shows the corresponding lateral wind speeds determining, at full scale, the same angles of the relative velocity of the wind considered in the wind tunnel tests.

The wind tunnel tests and the full scale operating conditions are different since, in the wind tunnel tests, both the barrier and the train are constrained to the ground, i.e. there is not relative motion. Therefore the pressure data, measured on the wall at different locations along the direction of the train axis, are, at full scale, representative of those measured at fixed points at different instants of time during the train transit.

\subsection{Experimental results}

In order to compare the results obtained for different incoming wind velocities the pressure have been expressed in terms of pressure coefficients. The free stream velocity is adopted to compute the pressure coefficient $C_{P}$ related to each pressure tap: 


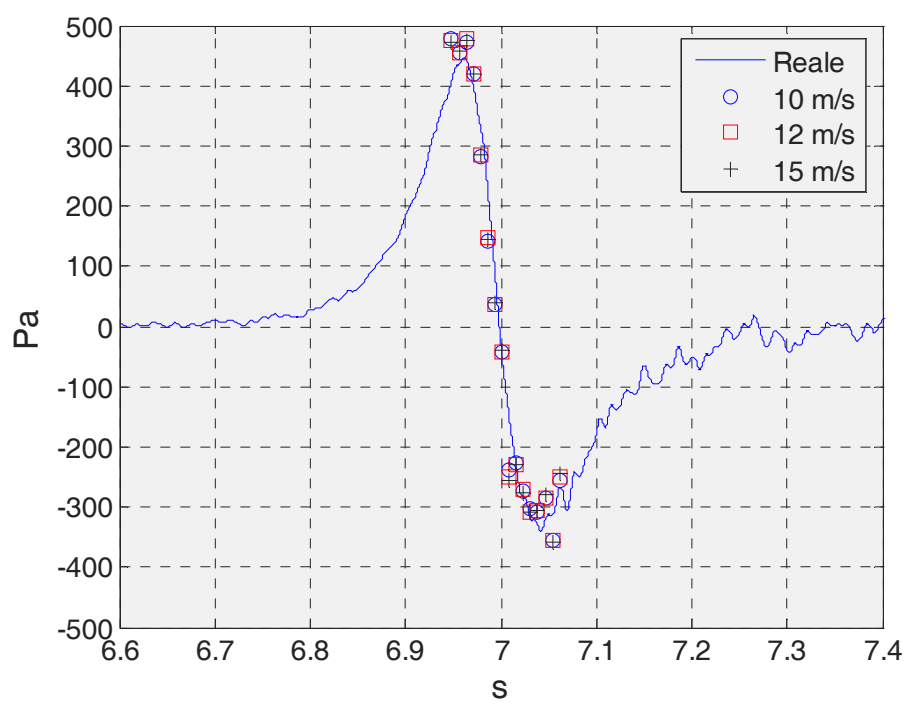

Figure 10: Comparison between wind tunnel tests and full scale measurement (1 m).

$$
C_{P}=\frac{2 p}{\rho V^{2}}
$$

where $p$ is the measured pressure, $\rho$ is the air density and $V$ is the wind free stream velocity. From the $C p$ values it is possible to define the pressure at the barrier as a function of the train speed. Using the following equation:

$$
p=\frac{1}{2} C_{P} \rho V_{T}^{2}
$$

and by considering the train velocity $V_{T}$ equal to $83 \mathrm{~m} / \mathrm{s}(300 \mathrm{~km} / \mathrm{h})$, a time distribution can be given to the wind tunnel data by means of the following equation:

$$
\Delta t=\frac{\Delta x}{V_{T}}
$$

being $\Delta \mathrm{t}$ is the time delay between two pressure taps and $\Delta \mathrm{x}$ is the distance between the same two pressure taps. So it is possible to compare the results obtained during wind tunnel tests with the results obtained at full scale on the high-speed line close to Anagni-Fiuggi, as shown in Figure 10, where a very good agreement can be seen. The comparison between wind tunnel results and CFD ones highlights a good agreement on the values of the positive peak, while the numerical approach overestimates the negative peak values, possibly due to the very simplified train geometry used for the simulations.

Figure 10 shows the values obtained at the height of $1 \mathrm{~m}$, full scale, while Figure 11 and Figure 13 are concerning, respectively, to $2 \mathrm{~m}$ and $3 \mathrm{~m}$. 


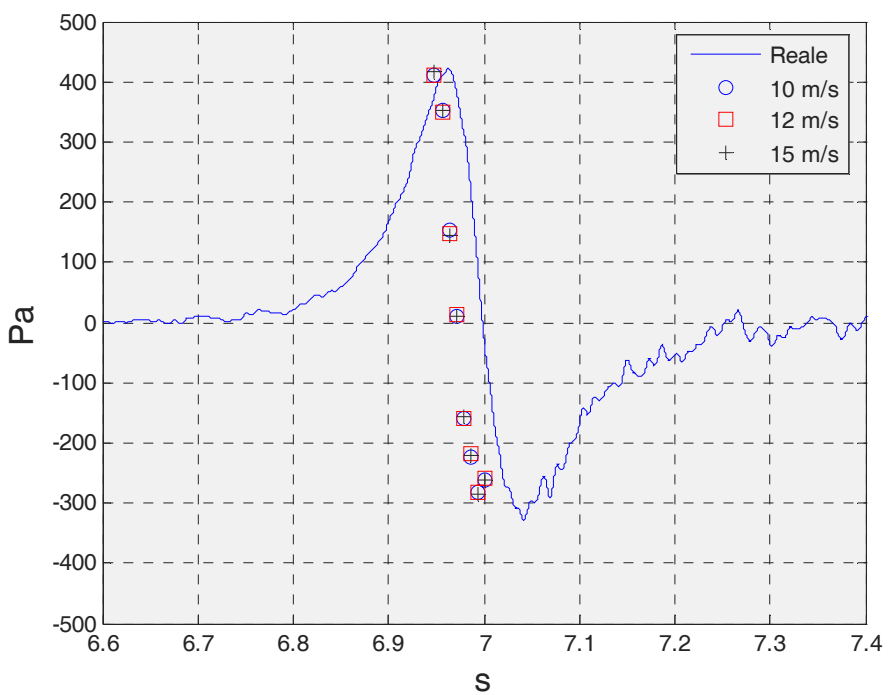

Figure 11: Comparison between wind tunnel test and full-scale measurements $(2 \mathrm{~m})$.

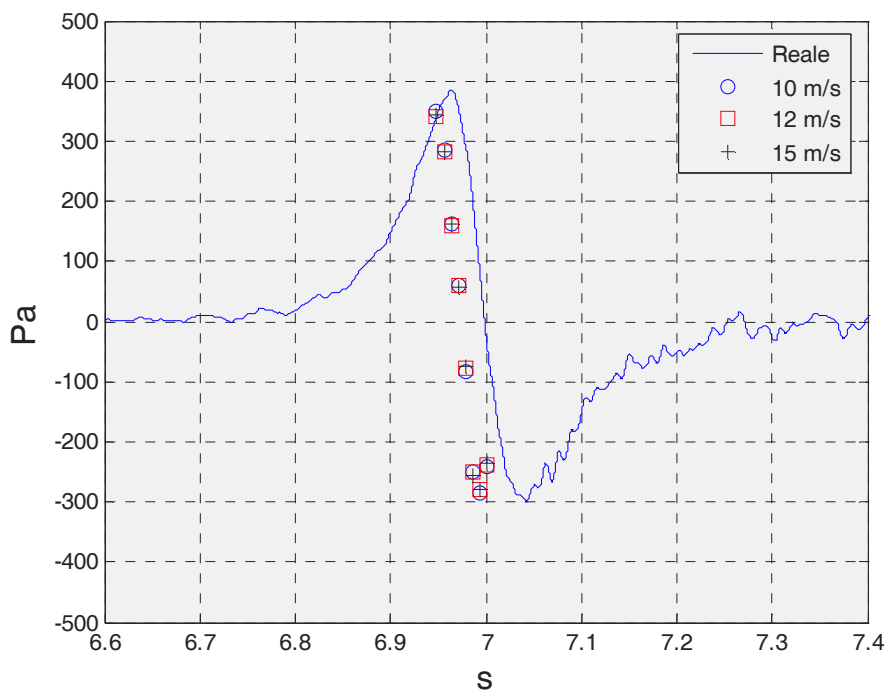

Figure 12: Comparison among wind tunnel tests and full-scale measurements $(3 \mathrm{~m})$.

The peak-to-peak amplitude of the pressure decreases with the height, both in full scale and in wind tunnel tests, as already explained by means of the CFD code. It is noticeable that there is a time shift between the laboratory conditions and the real condition data. This could be due to the different shape of the real train and the 1:10 scale model, particularly to the difference in the nose of the real train compared to the model's one. 


\subsection{Lateral wind}

Lateral wind effect has been studied by changing the yaw angle of the whole scenario by means of the turntable. There are no reference data with lateral wind at full scale, so the laboratory findings have been compared to no wind conditions, see Figure 13. The data are reported as a function of time and, in order to extend the results to the full-scale values, the reference velocity has been calculated as:

$$
V_{a}=\sqrt{V_{t}^{2}+V_{l a t}^{2}}
$$

where $V_{a}$ is the reference velocity, $V_{t}$ is the train speed and $V_{\text {lat }}$ is lateral wind velocity. The lateral wind has the effect to increase the pressure peaks, as already pointed out by the analysis of the CFD results.

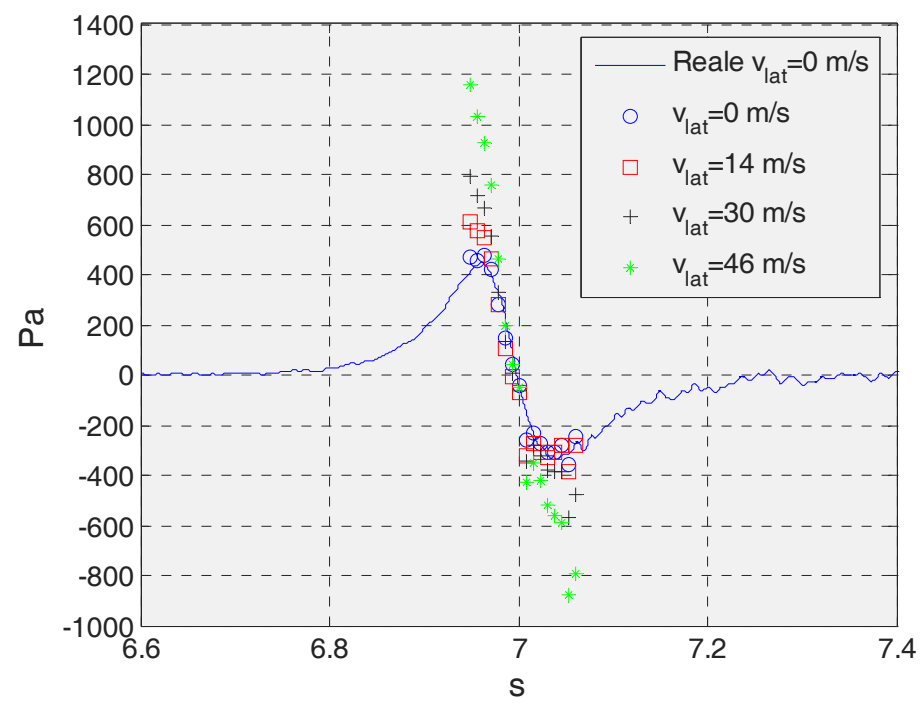

Figure 13: Comparison between wind tunnel and full-scale measurements, lateral wind effects at $\mathrm{h}=1 \mathrm{~m}$.

\section{Conclusions}

The pressure distribution due to a train transit on a noise reduction barrier has been investigated through CFD simulations, wind tunnel tests and full-scale measurements. Full-scale measurements are the more reliable data but, with this approach, it is difficult to take into account the lateral wind effects. CFD analysis is able to consider both lateral wind and no lateral wind conditions and allows one to have a good insight in the phenomena. The strong simplification in the train geometry may be the cause of the overestimated negative pressure peak 
obtained by the CFD results. Wind tunnel tests on a still train model proved to be a valuable tool to investigate the pressure distribution not only in absence of lateral wind. Even though in the wind tunnel experiments there is not relative motion between the train and the scenario and the wind-train velocity composition is not reproduced, the wind tunnel test data highlight the effects of the lateral wind on the values of the negative pressure peak.

\section{References}

[1] Copley, J.M., 1987. The three-dimensional flow around railway trains. Journal of Wind Engineering and Industrial Aerodynamics, 26(1), pp. 21-52.

[2] Barcala, M.A. and Meseguer, J., 2007. An experimental study of the influence of parapets on the aerodynamic loads under cross wind on a twodimensional model of a railway vehicle on a bridge. Proceedings of the Institution of Mechanical Engineers, Part F: Journal of Rail and Rapid Transit, 221(4), pp. 487-494.

[3] Papesch, A.J.G., 1972. Model Study of Windbreaks on Railway Bridges. New Zealand Engineering, 27(4), pp. 132-139.

[4] Tanemoto, K., 2004. Wind tunnel tests on aerodynamic characteristics of train/vehicles in cross winds and effects of wind-protection fences. Railway Technical Research Institute Report, 18(9), pp. 17-22. 